\title{
Infección del torrente circulatorio en una unidad de neonatología de Medellín-Colombia, 2008-2009
}

\author{
Alvaro Hoyos O., Margarita Suarez G., Mónica Massaro C., \\ Gloria Ortiz M., José Aguirre C. y Andrés Uribe M.
}

\section{Bloodstream infection in a neonatology unit of Medellín-Colombia, 2008-2009}

Bloodstream infection (BSI) is one of the main causes of sepsis and death in newborns. The relative importance of nosocomial and non-nosocomial agents in developing countries is not clear. We conducted a prospective study of newborns hospitalized with a first episode of BSI in order to classify it as early, late or nosocomial, describe the clinical and microbiological charateristics, and explore risk factors between hospital-acquired $v s$ community-acquiered BSI (HA-BSI vs CA-BSI). Twenty-seven newborns with a first episode of BSI were identified. Streptococcus agalactiae and coagulase negative Staphylococcus were the main agents in CA-BSI and HA-BSI, respectively. The only significant intrinsic risk factor between HA-BSI and CA-BSI was gestational age. The frequent finding of $S$. agalactiae warrants routine screening and prophylaxis in pregnant women. The risk factors for HA-BSI were mostly extrinsic, and thus, susceptible of being modified..

Key words: Bacteremia, microbiology, risk factors, newborn.

Palabras clave: Bacteriemia, microbiología, factores de riesgo, recién nacido.

\section{Introducción}

S e estima que las infecciones neonatales causan 1,6 millones de muertes anuales. En países en vía de desarrollo, estas infecciones son responsables de $\sim 40 \%$ de los 4 millones de muertes en niños bajo 30 días de vida. Sin embargo, el porcentaje de mortalidad varía entre 4 y $56 \%$ según datos de estudios hospitalarios y entre 8 y $80 \%$ en estudios poblacionales ${ }^{1-5}$.

La infección del torrente circulatorio (ITC) o bacteriemia se define como el aislamiento de microorganismos patógenos o potencialmente patógenos en uno o más cultivos de sangre, que pudiera o no estar relacionada con un foco infeccioso ${ }^{4,6}$. Este tipo de infección es una de las enfermedades más serias y potencialmente fatales descritas en niños y la principal causa de sepsis neonatal ${ }^{1}$. La tasa de ITC varía entre 1,7 y 33 casos por cada 1.000 recién nacidos, siendo África y Asia los lugares que reportan cifras más elevadas ${ }^{1}$. Estas tasas son 3 a 30 veces más altas que la de 1 a 5 por cada 1.000 recién nacidos reportadas en países industrializados ${ }^{1}$. La etiología de la sepsis neonatal de la comunidad en países en vía de desarrollo no está suficientemente esclarecida ${ }^{7}$.

En Colombia son pocos los estudios publicados sobre sepsis neonatal ${ }^{8-11}$ y son aún más escasos los relacionados exclusivamente al tema de la ITC ${ }^{12,13}$. Debido a lo anterior, se realizó un estudio en una institución de alto nivel de complejidad, en neonatos hospitalizados con ITC cuyos objetivos fueron: describir las características clínicas y microbiológicas en esta cohorte de pacientes, clasificar la ITC de acuerdo al momento de su adquisición como de la comunidad (ITC-AC, temprana y tardía) o nosocomial (adquirida en el hospital, ITC-AH) y explorar posibles factores de riesgo entre las ITC-AC y las ITC-AH.

\section{Materiales y Métodos}

Estudio prospectivo, realizado en la cohorte de neonatos con ITC entre el $1^{\circ}$ de marzo del 2008 y 30 de abril de 2009. El estudio se realizó en la Clínica Universitaria Bolivariana (CUB), institución de alto nivel de complejidad en neonatología. Dicha institución cuenta con una unidad de recién nacidos de 25 camas conformadas por tres salas de hospitalización separadas la una de la otra: cuidados intensivos neonatales, intermedios y una sala de recuperación nutricional o cuidados básicos. El estudio contó con la aprobación del Comité de Investigación y Ética de la CUB.

Criterios de inclusión y de exclusión. Se incluyeron todos los pacientes $\leq 30$ días de vida al ingreso, hospitalizados en el Servicio de Neonatología que presentaron un primer cultivo de sangre positivo (episodio de bacteriemia) tomado por vena periférica y/o por catéter central (CC) arterial o venoso. Se excluyeron los neonatos con

\author{
Clínica Universitaria \\ Bolivariana, Medellín, \\ Colombia (AHO, GOM, JAC, \\ AUM). \\ Facultad de Medicina, \\ Universidad Pontificia \\ Bolivariana. (MSG, MMC). \\ Estudio realizado en la Clínica \\ Universitaria Bolivariana, Servicio de \\ Pediatría, Unidad de Neonatología. \\ Fuente de financiación: Fondo \\ para la investigación de la Clínica \\ Universitaria Bolivariana. \\ Los autores declaran no tener \\ conflictos de intereses. \\ Recibido: 22 de abril de 2010 \\ Aceptado: 22 de septiembre de \\ 2010 \\ Correspondencia a: \\ Álvaro Hoyos Orrego \\ alvaromicro@hotmail.com
}


un cultivo positivo diferente a la sangre (por ejemplo, urocultivo) pero con hemocultivos negativos. También se excluyeron los pacientes que presentaron un solo hemocultivo positivo para Staphylococcus coagulasa negativa y que mejoraron con un tratamiento no efectivo para este microorganismo. En este último caso, el resultado del hemocultivo se interpretó como contaminación y se excluyó del análisis.

Recolección de la información. Identificado un paciente con diagnóstico clínico de sepsis neonatal se realizó seguimiento a los hemocultivos. Una vez que estos fueron positivos, e identificado el agente responsable, uno de los investigadores procedió a la recolección de los siguientes datos: demográficos, características clínicas al nacimiento, información microbiológica, factores de riesgo previo a la ITC y diagnósticos asociados al momento de la bacteriemia.

Definiciones operativas. Se clasificó a las ITC de acuerdo al origen de su adquisición, como provenientes de la comunidad ITC-AC o nosocomial ITC-AH. Para clasificar la ITC como adquirida en la comunidad se tuvo en cuenta que los pacientes no presentaran antecedente de exposición al ambiente hospitalario diferente al de la atención del parto (lo cual usualmente refleja transmisión vertical) ${ }^{4}$. La ITC-AC temprana se definió como la bacteriemia que ocurrió en las primeras 72 horas de vida y la ITC-AC tardía como la bacteriemia que ocurrió a partir de 72 horas de vida ${ }^{4}$. Se consideró ITC-AH aquella que ocurrió $\geq 72$ horas de estancia hospitalaria en pacientes que no tuvieran evidencia de infección al momento del ingreso, de acuerdo a la definición propuesta por Efird $\mathrm{MM}^{10}$ y Rojas $\mathrm{MA}^{11}$ y a la modificada de Haque $\mathrm{KN}^{4}$. Para el diagnóstico de bacteriemia asociada a catéter se utilizó el criterio de tiempo diferencial según las recomendaciones publicadas ${ }^{14,15}$.

Como co-morbilidades sépticas, previas o posteriores al diagnóstico de ITC, se consideraron: neumonía, infección urinaria, meningitis, enterocolitis y trombocitopenia, con base en los resultados de exámenes de laboratorio (radiografía de tórax o abdomen, urocultivo, cultivo de líquido cefalorraquídeo -LCR-, hemograma, entre otros) y del criterio del médico tratante, de acuerdo a las guías de manejo de la institución.

La ITC primaria y secundaria se definieron como la bacteriemia sin un foco de infección identificable o la causada por un patógeno relacionado con infección en otro sitio (por ejemplo neumonía), respectivamente ${ }^{4}$.

Protocolo de toma de hemocultivos: Se empleó la técnica de venopunción en al menos dos sitios diferentes, con asepsia previa con clorhexidina y diferencia de tiempo entre muestras de 20 minutos. El volumen de sangre inoculada para cada una de las botellas fue de 1-3 mL (set de hemocultivos).

Microbiología. Los hemocultivos fueron llevados al laboratorio de microbiología y se procesaron utilizando el equipo Bactec 9120 (Becton Dickinson $^{\mathrm{TM}}$, Sparks, Maryland, USA). Este equipo permite calcular el tiempo diferencial de crecimiento microbiano entre las dos muestras en los casos en que se tomaron de manera simultánea un hemocultivo a través de CC y uno por vena periférica. La identificación de la bacteria, las pruebas de susceptibilidad a los antimicrobianos y la presencia de $\beta$-lactamasas de espectro extendido (BLEE) o de $\beta$-lactamasas tipo AmpC (presuntivo) se realizaron utilizando el equipo automatizado Phoenix 100 (Becton Dickinson $^{\mathrm{TM}}$, Sparks, Maryland, USA).

Análisis estadístico: Se realizó análisis univariado y bivariado. Para las variables cuantitativas se utilizaron medidas de tendencia central (promedio y desviación estándar [DE]) y para las variables cualitativas, distribución de frecuencias. Para la comparación de medias y de proporciones, entre ITC-AC (temprana y tardía) e ITC-AH, se utilizaron las pruebas de Mann-Whitney y Fisher (prueba exacta), respectivamente, considerando un valor $\mathrm{p}<0,05$ como estadísticamente significante. La base de datos y el análisis estadístico se desarrolló en los programas Excel, Epi Info 6.04 y SPSS 12.0.

\section{Resultados}

En un período de 14 meses se procesaron 541 sets de hemocultivos en neonatos hospitalizados, de los cuales 27 correspondían a un primer episodio de ITC; teniendo en cuenta que el número de egresos del servicio de neonatología durante el período de estudio fue de 1.058 pacientes, la incidencia de ITC fue de 2,55\%. La relación por género (hombre: mujer) fue de 1,5:1,0 predominando los recién nacidos pretérmino (RNPT) con un peso inferior a $2.500 \mathrm{~g}$. Las características al nacimiento se muestran en la Tabla 1.

Se aislaron 28 microorganismos de 27 neonatos (en un paciente se aislaron dos agentes en una misma muestra de hemocultivos). La distribución fue la siguiente: cocáceas grampositivas (n: 17), bacilos gramnegativos fermentadores (n: 8) y bacilos gramnegativos no fermentadores (n: 3) (Tabla 2). En cuanto a los agentes aislados y su relación con el tiempo de presentación de la ITC, la mayoría de infecciones tempranas como tardías de la comunidad fueron causadas por especies de los géneros Streptococcus y Staphylococcus. Los dos pacientes que presentaron bacteriemia tardía de la comunidad no tenían antecedente de hospitalización previa al momento del inicio de la sintomatología e ingresaron al servicio como 
Tabla 1. Características al nacimiento y factores de riesgo en 27 neonatos con infección del torrente circulatorio ${ }^{a}$

\begin{tabular}{|c|c|}
\hline Características al nacimiento & $n=27(\%)$ \\
\hline Género masculino & $16 \quad(59)$ \\
\hline RNPT & $19(70)$ \\
\hline RNT & $8(29,6)$ \\
\hline \multicolumn{2}{|l|}{ Peso en gramos } \\
\hline$\leq 1.000$ & $7 \quad(26)$ \\
\hline $1.001-1.500$ & $3(11)$ \\
\hline $1.501-2.000$ & $5(18,5)$ \\
\hline $2.001-2.500$ & $3(11)$ \\
\hline $2.501-3.500$ & $8(29,6)$ \\
\hline$>3.501$ & $1 \quad(3,7)$ \\
\hline PAEG & $17 \quad(63)$ \\
\hline PBEG & $10 \quad(37)$ \\
\hline \multicolumn{2}{|l|}{ Diagnósticos al ingreso } \\
\hline Riesgo séptico & $12(44,4)$ \\
\hline Riesgo metabólico & $13(48)$ \\
\hline Riesgo respiratorio & $14 \quad(52)$ \\
\hline RCIU & $4(15)$ \\
\hline Dismorfismos y malformaciones & $6(22)$ \\
\hline Otros diagnósticos ${ }^{\mathrm{b}}$ & $6 \quad(22)$ \\
\hline
\end{tabular}

aAlgunas características al nacimiento pudieran ser también factores de riesgo para ITC. 'Otros diagnósticos como: síndrome de aspiración de meconio, mala adaptación neonatal, riesgo de síndrome de abstinencia, riesgo social, entre otros. ITC: infección del torrente circulatorio; RNPT: recién nacido pretérmino; RNT: recién nacido de término; PAEG: peso adecuado para la edad gestacional; PBEG: peso bajo para la edad gestacional; RCIU: retraso del crecimiento intrauterino. casos probables de sepsis. En los casos de ITC-AH predominó Staphylococcus coagulasa negativa (Tabla 3).

Susceptibilidad in vitro. Todos los estreptococos fueron sensibles a penicilina, los estafilococos coagulasa negativa fueron resistentes a oxacilina pero sensibles a vancomicina, Staphylococcus aureus fue sensible a oxacilina y dos aislados de Enterococcus faecalis fueron sensibles a ampicilina. Pseudomonas aeruginosa y Acinetobacter baumannii fueron multisensibles (susceptibilidad a tres o más diferentes clases de antimicrobianos) al igual que Escherichia coli, Klebsiella oxytoca y la mayoría de las Klebsiella pneumoniae. Sólo se aisló un bacilo gramnegativo ( $K$. pneumoniae) BLEE positivo y dos Serratia marcescens productoras, presuntivamente, de $\beta$-lactamasa tipo AmpC, ya que mostraban resistencia a cefoxitina y sulbactam/ampicilina (Tabla 4).

Como co-morbilidades, previas o posteriores al diagnóstico de ITC se encontraron las siguientes: apnea (n: 7); trombocitopenia (n: 6); meningitis (n: 3); neumonía (n: 4); enterocolitis necrosante (n: 3 ); infección del tracto urinario (ITU) (n: 3); coagulación intravascular diseminada (n: 1). En dos de los tres casos de meningitis se aisló del LCR: S. epidermidis y Streptococus bovis II, respectivamente; en un solo caso, el diagnóstico de meningitis se realizó por parámetros indirectos a través del citoquímico y diferencial de células en el LCR. En los casos de ITU, los aislados correspondieron a K. pneumoniae, E. faecalis y Staphylococcus sciuri.

No se presentaron casos, que por definición, cumplieran criterios para bacteriemia asociada a catéter.

$\mathrm{Al}$ realizar el análisis exploratorio de los posibles

Tabla 2. Tipos de microorganismos aislados en los hemocultivos de 27 neonatos

\begin{tabular}{|c|c|c|c|c|c|}
\hline \multirow{2}{*}{$\begin{array}{l}\text { Cocáceas grampositivas } \\
\text { (n: 17) } \\
\text { Staphylococcus hominis }\end{array}$} & \multirow[b]{2}{*}{1} & \multicolumn{2}{|c|}{$\begin{array}{l}\text { Bacilos gramnegativos fermentadores } \\
\qquad(\mathrm{n}: 8)\end{array}$} & \multicolumn{2}{|c|}{$\begin{array}{l}\text { Bacilos gramnegativos no fermentadores } \\
\qquad(\mathrm{n}: 3)\end{array}$} \\
\hline & & Klebsiella pneumoniae & 4 & Pseudomonas aeruginosa & 1 \\
\hline S. epidermidis & 4 & K. oxytoca & 1 & Acinetobacter baumannii & 1 \\
\hline S. xylosus & 1 & Escherichia coli & 1 & Chryseobacterium meningosepticum & 1 \\
\hline S. sciuri & 1 & \multirow[t]{7}{*}{ Serratia marcescens } & \multirow[t]{7}{*}{2} & & \\
\hline S. cohnii spp cohnii & 1 & & & & \\
\hline S. aureus & 1 & & & & \\
\hline Streptococcus agalactiae & 4 & & & & \\
\hline S.bovis II & 1 & & & & \\
\hline Streptococcus sp & 1 & & & & \\
\hline Enterococcus faecalis & 2 & & & & \\
\hline
\end{tabular}


Tabla 3. Microorganismos aislados y su relación con la infección del torrente circulatorio de presentación temprana, tardía y nosocomial

\begin{tabular}{|c|c|c|c|}
\hline \multirow[t]{2}{*}{ Microorganismo } & \multicolumn{2}{|c|}{ ITC-AC } & \multirow{2}{*}{$\begin{array}{c}\text { ITC-AH } \\
\left(\mathrm{n}=18^{\mathrm{a}}\right)\end{array}$} \\
\hline & $\begin{array}{c}\text { ITC temprana } \\
(n=7)\end{array}$ & $\begin{array}{c}\text { ITC tardía } \\
(\mathrm{n}=2)\end{array}$ & \\
\hline S. epidermidis & & & 4 \\
\hline S. hominis & & & 1 \\
\hline S. xylosus & & & 1 \\
\hline S. sciuri & & 1 & \\
\hline S. cohnii spp cohnii & & & 1 \\
\hline S. aureus & 1 & & \\
\hline S. agalactiae & 3 & 1 & \\
\hline S. bovis II & & & 1 \\
\hline Streptococcus sp & 1 & & \\
\hline E. faecalis & & & 2 \\
\hline K. pneumoniae & & & 4 \\
\hline K. oxytoca & & & 1 \\
\hline E. coli & 1 & & \\
\hline S. marcescens & & & 2 \\
\hline$P$. aeruginosa & 1 & & \\
\hline A. baumannii & & & 1 \\
\hline C. meningosepticum & & & 1 \\
\hline Total $=28$ & $7(25 \%)$ & $2(7,1 \%)$ & $19^{\mathrm{a}}(67,8 \%)$ \\
\hline
\end{tabular}

factores de riesgo intrínsecos se encontró una menor edad gestacional en el grupo de ITC-AH (n: 18) comparado con el de ITC-AC (n: 9), siendo esta diferencia estadísticamente significativa (Tabla 5). No se encontró diferencia entre los grupos para las variables: ruptura de membranas ovulares (RMO) ni peso al nacimiento (Tabla 5). Para la ITC-AH, la frecuencia de exposición a los factores de riesgo extrínsecos fue: nutrición parenteral total [NPT] $(83,3 \%)$, sonda oro-gástrica $(83,3 \%)$, antimicrobianos previos $(66,6 \%)$, uso de catéter epicutáneo $(66,6 \%)$, antiácidos $(66,6 \%)$, ventilación mecánica $(50 \%)$, uso de CC $(44,4 \%)$, transfusiones $(44,4 \%)$ y uso de catéter vesical $(16,6 \%)$. El número de días promedio de uso de dispositivos en el grupo de ITC-AH fue: 13 para el epicutáneo, 7,25 para CC, 7,11 para ventilación mecánica y 2,5 para catéter vesical.

\section{Discusión}

En nuestro conocimiento este es el primer estudio a nivel local que de manera prospectiva clasifica la ITC neonatal en adquiridas en la comunidad (temprana y tardía) y nosocomial; además, discrimina la etiología según el momento de aparición de la bacteriemia y compara los factores de riesgo intrínsecos entre eventos no nosocomiales y nosocomiales. En adición, los hallazgos en la microbiología difieren en parte con lo reportado en países en vía de desarrollo ${ }^{1,7,16}$.

Los microorganismos predominantes aislados en los hemocultivos fueron cocáceas grampositivas entre los cuales se destacan los estreptococos (principalmente $S$.

Tabla 4. Microorganismos aislados y su resistencia frente a algunos antimicrobianos

\begin{tabular}{|c|c|c|c|c|c|c|c|c|c|c|c|c|c|c|c|}
\hline Microorganismo & ox & cC & SXT & $\mathbf{E}$ & ATM & AN & S-AM & Fox & TZP & $\mathbf{R A}$ & FEP & MEP-IPM & CTX & $\beta$-L AmpC & $\beta$ LEE \\
\hline S. epidermidis ${ }^{\mathrm{a}}$ & 4 & 4 & 3 & 4 & & & & & & 1 & & & & & \\
\hline S. hominis & 1 & & 1 & & & & & & & & & & & & \\
\hline S. $x y l o s u s^{a}$ & 1 & 1 & 1 & 1 & & & & & & & & & & & \\
\hline S. sciuria & 1 & 1 & & & & & & & & 1 & & & & & \\
\hline S. cohnii spp cohnii & 1 & & & & & & & & & & & & & & \\
\hline S. bovis II & & 1 & & 1 & & & & & & & & & & & \\
\hline K. pneumoniae & & & & & 1 & & 1 & & & & 1 & & 1 & & 1 \\
\hline E. coli & & & 1 & & & & & & & & & & & & \\
\hline S. marcescens & & & & & & 1 & 2 & 2 & 1 & & & & & 2 & \\
\hline C. meningosepticum & & & & & 1 & 1 & & & & & 1 & 1 & 1 & & \\
\hline
\end{tabular}




\begin{tabular}{|c|c|c|c|c|c|c|c|}
\hline \multirow[t]{2}{*}{ Variables } & \multicolumn{3}{|c|}{ ITC-AC } & \multicolumn{3}{|c|}{ ITC-AH } & \multirow[b]{2}{*}{ Valor $\mathbf{p}$} \\
\hline & $n=9(\%)$ & $\bar{x}$ & DE & $n=18(\%)$ & $\bar{x}$ & DE & \\
\hline $\mathrm{RMO}^{\mathrm{a}, \mathrm{b}}$ & $3 / 8(37,5)$ & & & $6 / 17(35,2)$ & & & 0,734 \\
\hline Peso $\leq 2.500 \mathrm{~g}^{\mathrm{b}}$ & $4 / 9(44,4)$ & & & $14 / 18(77,8)$ & & & 0,090 \\
\hline Peso promedio en $\mathrm{g}$ al nacimiento ${ }^{c}$ & & 2.423 & 840 & & 1.713 & 863 & 0,085 \\
\hline Edad gestacional $\leq 36$ semanas $^{b}$ & $4 / 9(44,4)$ & & & $16 / 18(88,9)$ & & & 0,020 \\
\hline \multicolumn{2}{|l|}{ Edad gestacional promedio en semanasc } & 36 & 3,7 & & 32 & 3,9 & 0,020 \\
\hline \multicolumn{8}{|c|}{$\begin{array}{l}\text { aEn un caso de ITC temprana y uno de ITC tardía no se determinó el tiempo de ruptura de membranas. 'Comparación de proporciones a travé } \\
\text { de la prueba exacta de Fisher. 'CComparación de medias (prueba de Mann-Whitney). ITC-AC: infección del torrente circulatorio adquirida en I } \\
\text { comunidad; ITC-AH: adquirida en el hospital; RMO: ruptura de membranas ovulares; DE: desviación estándar; } \bar{x} \text { : promedio. }\end{array}$} \\
\hline
\end{tabular}

agalactiae) y estafilococos coagulasa negativa; entre los bacilos gramnegativos fermentadores, Klebsiella spp fue el aislado más frecuente. Lo anterior difiere de lo reportado en la literatura médica en que los bacilos gramnegativos entéricos y ambientales son la causa predominante de bacteriemia en países en vía de desarrollo, mientras que en países desarrollados los patógenos principales son $S$. agalactiae, E. coli y Staphylococcus coagulasa negativa ${ }^{1,16-18}$.

Al discriminar los agentes etiológicos de acuerdo al tiempo de aparición de la ITC, se aprecia que $S$. agalactiae predominó en la bacteriemia temprana y tardía de la comunidad (n: 4) y Staphylococcus coagulasa negativa (n: 7) y otras cocáceas grampositivas en la nosocomial; $K$. pneumoniae, $K$. oxytoca y $S$. marcescens fueron los bacilos gramnegativos más frecuentes en la ITC-AH (n: 7). Son pocos los estudios que reportan la etiología de la ITC neonatal según el tiempo de aparición; por ejemplo, Uddin Ahmed y cols publicaron un estudio sobre septicemia neonatal en un hospital de tercer nivel en la ciudad de Dhaka, Bangladesh, y encontraron que la mayoría de los agentes responsables de la bacteriemia temprana $(\leq$ 7 días) y tardía ( $>7$ días) fueron $E$. coli, $K$. pneumoniae y $S$. aureus $^{16}$. Recientemente, Zaidi y cols publicaron una revisión de estudios (oriente medio y Asia central, sureste asiático y pacífico, Africa y América Latina) enfocados a la etiología de la infección neonatal grave (bacteriemia, neumonía, meningitis) de la comunidad en la primera semana de vida y luego de la primera semana hasta los 28 días. En la primera semana, Klebsiella spp, $E$. coli y $S$. aureus fueron los principales patógenos; $S$. agalactiae fue infrecuente pero con variaciones entre regiones. Después de la primera semana de vida, $S$. aureus, $S$. agalactiae y Salmonella spp no tífica fueron los patógenos más comunes ${ }^{7}$. Los autores de este último trabajo destacan lo limitado de la información, la falta de criterios unificados en el diagnóstico de sepsis temprana y tardía, de la comunidad y hospitalaria y la presencia de agentes ambientales que pudieran sugerir que la infección ocurrió en el ambiente domiciliario, donde el parto tuvo lugar en muchas ocasiones (pobres prácticas higiénicas), más que por una probable transmisión vertical. Las variaciones regionales en incidencia e importancia relativa de $S$. agalactiae no están claramente dilucidadas; pudieran contribuir factores de riesgo como colonización vaginal, virulencia de las cepas, presencia de anticuerpos maternos o prácticas culturales ${ }^{8,16}$. Algunos trabajos locales como el de Efird y cols, en un estudio multicéntrico colombiano sobre la epidemiología de la infección nosocomial en unidades de cuidados intensivos neonatales, reportaron un predominio de bacilos gramnegativos sobre los grampositivos ( 55 vs $38 \%$ ) en pacientes $<2.000 \mathrm{~g}$, siendo la ITC el sitio más frecuente de infección. De forma individual, el patógeno más común fue Staphylococcus coagulasa negativa seguido de $K$. pneumoniae ${ }^{10}$. Eraso y cols publicaron un estudio epidemiológico de la sepsis nosocomial en la unidad de recién nacidos de la Fundación Cardio Infantil de Bogotá, Colombia, en que los bacilos gramnegativos $(71,2 \%)$, principalmente $K$. pneumoniae, fueron los agentes más comúnmente aislados, seguido de cocáceas grampositivas $(19,2 \%)$ y hongos $(9,6 \%)$. El sitio más frecuente de aislamientos fue la sangre ${ }^{9}$. Al comparar los hallazgos de estos dos últimos estudios con lo encontrado en la presente investigación, se aprecia la divergencia etiológica que puede existir entre instituciones a nivel local.

Al evaluar el perfil de resistencia de los diferentes microorganismos se aprecia que todos los estafilococos coagulasa negativa fueron resistentes a oxacilina pero sensibles a vancomicina. Esto último era lo esperado, ya que la gran mayoría de ITC por este agente fueron nosocomiales. Cifuentes y cols, en un estudio del perfil microbiológico de aislados en una unidad neonatal de Bogotá, Colombia, reportaron que $86,4 \%$ de los estafilo- 
cocos coagulasa negativa fueron resistentes a oxacilina y $99,4 \%$ fueron sensibles a vancomicina ${ }^{8}$. Eraso y cols, en otro grupo de neonatos de esta misma ciudad reportaron que los estafilococos coagulasa negativa aislados (n: 11) fueron resistentes a oxacilina pero todos sensibles a vancomicina ${ }^{9}$.

Fueron muy pocos los bacilos gramnegativos fermentadores y no fermentadores aislados en el presente trabajo, lo cual no permite hacer comparaciones con otros reportes locales; sin embargo, sólo se encontró una bacteria productora de BLEE (K. pneumoniae) y una bacteria presuntamente productora de $\beta$-lactamasas de tipo $\operatorname{AmpC}(S$. marcescens), sin el uso previo de cefalosporinas de tercera generación o aztreonam. Klebsiella pneumoniae $\mathrm{BLEE}+$, S. marcescens AmpC + , P. aeruginosa, A. baumannii y Chryseobacterium meningosepticum son aislados típicos en pacientes de las unidades de cuidados intensivos y sugieren que fueron colonizados rápidamente luego de su ingreso a la unidad neonatal y previo a la ITC.

Las co-morbilidades encontradas en esta cohorte de neonatos hacen referencia a las patologías que pudieran tener relación directa o indirecta con la ITC. En el caso de meningitis e ITU, éstas pueden haber sido secundarias a bacteriemias primarias con siembras a distancia. Por el contrario, en los casos de enterocolitis y neumonía se pudieron originar bacteriemias secundarias a traslocación bacteriana $^{19}$.

La ausencia de bacteriemia asociada a CC puede explicarse en parte por el tipo de catéter epicutáneo utilizado (el más frecuente de los CCs), el cual al ser de un calibre muy delgado no permitió, en muchos casos, obtener muestras de sangre para hemocultivos (retorno negativo), lo que imposibilitó la aplicación del criterio de tiempo diferencial establecido para su diagnóstico ${ }^{14,15}$.

Los factores de riesgo reportados en la literatura médica para sepsis neonatal no difieren de los encontrados para el grupo de ITC-AH en esta cohorte de pacientes ${ }^{11,19-23}$. Sin embargo, en el presente trabajo sólo se incluyeron pacientes infectados con hemocultivos positivos y no todos los casos de sepsis. En Colombia, Del Río y cols encontraron una asociación entre nutrición parenteral total (NPT) y la contaminación en su preparación con la presencia de bacteriemia secundaria por Enterobacter spp y E. coli ${ }^{12}$. En otro estudio, Hoyos y cols estudiaron la bacteriemia por $K$. pneumoniae en un grupo de neonatos encontrando un predominio de cepas multi-resistentes BLEE positivas sin el uso previo de cefalosporinas de tercera generación $\mathrm{u}$ otros antimicrobianos inductores de estas enzimas; en dicho estudio, los factores de riesgo descritos fueron el uso de CCs incluyendo el epicutáneo y las transfusiones de productos sanguíneos, entre otros ${ }^{13}$.

En el presente estudio, al comparar los factores de riesgo intrínsecos, entre el grupo de neonatos con ITC-AC y el de ITC-AH, se destaca que la edad gestacional fue significativamente menor en los neonatos con ITC-AH; por el contrario, la RMO y el peso al nacimiento no presentaron diferencias significativas. Esto último coincide, en parte, con lo publicado por Rojas y cols en un estudio multicéntrico en Colombia sobre factores de riesgo para sepsis neonatal. Los autores no encontraron asociación entre el bajo peso al nacimiento y la edad gestacional, atribuyendo el hecho a la baja sobrevida de los recién nacidos pequeños e inmaduros en nuestro país ${ }^{11}$. Holmes y cols, en un estudio de factores de riesgo y vigilancia de ITC nosocomial, encontraron que 9 de los 11 factores intrínsecos y extrínsecos evaluados fueron asociados de manera significativa con ITC en el análisis univariado (excepto la lactancia materna y el género); sin embargo, en el modelo de regresión, los únicos factores de riesgo independientes fueron la NPT y la edad $<26$ semanas $(14,2 \text {, IC 8,8-22,9 y 2,5, IC 1,7-3,8, respectivamente })^{23}$.

En cuanto a los factores de riesgo extrínsecos y su relación con ITC-AH, en el caso de las transfusiones, es muy poco probable que haya ocurrido contaminación en los productos transfundidos ya que los casos de bacteriemia asociado a este procedimiento en pacientes pediátricos de otras salas son raros y una posible explicación pudiera ser una inadecuada manipulación y contaminación de los accesos vasculares a través de los cuales se infunden sangre y sus derivados ${ }^{13,24}$. En el caso de la NPT existe un riesgo para bacteriemia por Staphylococcus coagulasa negativa, mediado por el componente lípido de la preparación ${ }^{11}$; no es claro cómo la emulsión de lípidos predispone a la ITC pero se sugiere que hay un detrimento en la función de monocitos y neutrófilos e incremento en la colonización por Staphylococcus coagulasa negativa ${ }^{23}$. El uso de antimicrobianos previos puede predisponer a la colonización por microorganismos diferentes a los encontrados en los neonatos que no han recibido antimicrobianos $^{9}$; a su vez los antimicrobianos seleccionan la microbiota, de manera que predominan las bacterias resistentes sobre las sensibles. Por otra parte, la sonda oro-gástrica facilita la entrada y estimula el crecimiento de microorganismos del tracto gastrointestinal alto, además de lesionar directamente la mucosa y romper una barrera natural contra la infección ${ }^{11}$; de manera similar, las sondas naso-yeyunales evitan el efecto protector del ácido gástri$\mathrm{co}^{12}$. Adicionalmente, el uso de antiácidos incrementa el valor del $\mathrm{pH} \geq 4$ disminuyendo la capacidad proteolítica del jugo gástrico con la consecuente colonización del estómago por bacilos gramnegativos ${ }^{11}$. Por otro lado, los estafilococos coagulasa negativa exhiben una capacidad de colonización y formación de biopeliculas en los CCs, lo que permite que persista la infección; la refractariedad al efecto de los antimicrobianos en la biopelícula se debería a una combinación de factores como pobre penetración del fármaco, disminución de nutrientes, crecimiento lento, formación de múltiples capas y compromiso de las 
defensas del hospedero por la presencia de biomateriales ${ }^{8}$. Todos los factores de riesgo extrínsecos mencionados predominan, en el presente estudio, en los casos de ITC-AH, los cuales son susceptibles de modificar, a diferencia de algunos factores intrínsecos como la edad gestacional la cual dependería de situaciones materno fetales de difícil intervención.

Este estudio tiene limitaciones en cuanto a lo heterogéneo de los individuos, la falta de criterios unificados para algunos diagnósticos asociados y ciertas limitaciones en el laboratorio de microbiología. Además, la frecuencia observada de ITC y de los agentes aislados no permitió explorar asociación entre los factores de riesgo y microorganismos específicos.

La presencia de $S$. agalactiae como uno de los agentes principales en ITC-AC, permite plantear, a nivel institucional, la necesidad de implementar el cultivo de tamizaje a la madre gestante y la profilaxis periparto, lo que aún no sucede a la fecha. Además, los hallazgos en la microbiología y su perfil de susceptibilidad a los antimicrobianos sugieren que el cubrimiento empírico en la ITC temprana con ampicilina más un aminoglucósido continua siendo efectivo. En la ITC-AH predomina Staphylococcus coagulasa negativa con una frecuencia baja de bacilos gramnegativos multi-resistentes. A diferencia de algunos factores de riesgo intrínsecos para ITC que dependen de situaciones materno fetales de difícil intervención, la frecuencia de exposición a los factores de riesgo extrínsecos, en el caso de la ITC nosocomial, sí es susceptible de modificarse.
Agradecimientos: A todos los pediatras y profesionales de enfermería del Servicio de Neonatología; a la enfermera Aura María Gutiérrez, coordinadora del Comité de Vigilancia Epidemiológica; a los bacteriólogos de la sección de microbiología del laboratorio y al personal de archivo clínico de la CUB.

\section{Resumen}

La infección del torrente circulatorio (ITC) es una de las principales causas de sepsis y muerte neonatal. $\mathrm{Su}$ etiología en países en vía de desarrollo, entre agentes no nosocomiales vs nosocomiales no está suficientemente esclarecida. Estudio prospectivo que incluyó neonatos hospitalizados que presentaron un primer hemocultivo positivo, con el propósito de describir características clínicas, microbiológicas, clasificar la ITC en temprana, tardía y nosocomial, y explorar factores de riesgo entre ITC adquirida en la comunidad $v s$ ITC adquirida en el hospital (ITC-AC vs ITC-AH). Se identificaron 27 neonatos con un primer episodio de ITC. Streptococcus agalactiae y Staphylococcus coagulasa negativa fueron los principales agentes en ITC-AC e ITC-AH, respectivamente. El factor de riesgo intrínseco que mostró una diferencia significativa entre ITC-AC vs. ITC-AH fue la edad gestacional. La presencia de $S$. agalactiae permite plantear el tamizaje y profilaxis a la gestante. Los factores de riesgo para ITC-AH fueron en su mayoría extrínsecos, es decir, susceptibles de ser modificados.

\section{Referencias}

1.- Zaidi A, Charles W, Thaver D, Bhutta Z, Abbas Z, Goldmann D. Hospital-acquired neonatal infections in developing countries. Lancet 2005; 365: 1175-88.

2.- Thaver D, Zaidi A. Burden of neonatal infections in developing countries. A review of evidencia from community-based studies. Pediatr Infect Dis J 2009; 28: S3-S9.

3.- Qazi S and Stoll B. Neonatal sepsis. A major global public health challenge. Pediatr Infect Dis J 2009; 28: S1-S2.

4.- Haque K. Definitions of bloodstream infection in the newborn. Pediatr Crit Care Med 2005; 6: S45-9.

5.- Bahl R, Martínez J, Ali N, Bhan M, Carlo W, Chan K, et al. Research priorities to reduce global mortality from newborn infections by 2015. Pediatr Infect Dis J 2009; 28: S43-8.

6.- Kaplan S. Bacteremia and Septic Shock. Feigin R, Demmler G, Cherry J, Kaplan S, editors. Textbook of Pediatric Infectious Diseases, fifth ed. 2004. Philadelphia,
Pensylvania: Saunders. p. 810-24.

7.- Zaidi A, Thaver D, Ali S, Khan TA. Pathogens associated with sepsis in newborns and young infants in developing countries. Pediatr Infect Dis J 2009; 28:S10-8.

8.- Cifuentes Y, Ruiz A, Leal A, Muñoz L, Herrera M, Jiménez L. Perfil microbiológico de aislamientos en unidades neonatales en un hospital de tercer nivel de Bogotá, Colombia. Rev Salud Pública 2005; 7: 191-200.

9.- Eraso J, Troncoso G, Álvarez M, Ibáñez M. Comportamiento epidemiológico de la sepsis nosocomial en la unidad de recién nacidos de la fundación cardioinfantil. Rev Cienc Salud Bogotá (Colombia) 2008; 6: 36-49.

10.- Efird M, Rojas M, Lozano J, Bose C, Rojas M, Rondón M, et al. Epidemiology of nosocomial infections in selected neonatal intensive care units in Colombia, South America. J Perinatol 2005; 25: 531-6.

11.- Rojas M, Efird M, Lozano J, Bose C, Rojas M, Rondón M, et al. Risk factors for nosocomial infections in selected neonatal intensive care units in Colombia, South America. J Perinatol 2005; 25: 537-41.
12.- Del Río J, Jurado C, Arango F. Estudio de un brote de bacteriemia secundaria asociada con nutrición parenteral en una unidad de recién nacidos de tercer nivel. Colomb Med 1999; 30: 155-8.

13.- Hoyos A, Rivera O, Hoyos C, Mesa C, Alfaro J. Características clínicas, epidemiológicas y de susceptibilidad a los antibióticos en caso de bacteriemia por Klebsiella pneumoniae en neonatos. Rev CES Med 2007; 21: 17-25.

14.- Mermel L, Farr B, Sherertz R, Raad I, O'Grady N, Harris J, et al. Guidelines for the management of intravascular catheterrelated infections. Clin Infect Dis 2001; 32: 1249-72.

15.- Shah S, Smith M, Zaoutis T. Device-related infections in children. Pediatr Clin North Am 2005; 52: 1189-208.

16.- Nawshad A, Azad C, Hoque M, Darmstadt L. Clinical and bacteriological profile of neonatal septicemia in a tertiary level pediatric hospital in Bangladesh. Indian Pediatr 2002; 39: 1034-9.

17.- Molina J, Santana C, Hernández J, López I, Dorta E. Incidence of nosocomial infections 
at a neonatal intensive care unit: a six year surveillance study. Enferm Infecc Microbiol Clin 2006; 24: 307-12.

18.- Bem J, Bouziri A, Mnif K, Hamdi A, Khaldi A, Kchaaou W. Epidemiology of hospital-acquired bloodstream infections in a Tunisian pediatric intensive care unit: a 2-year prospective study. Am J Infect Control 2007; 35: 613-8.

19.- Perlman S, Saiman L, Larson E. Risk factors for late-onset health care-associated bloodstream infections in patients in neonatal intensive care units. Am J Infect Control 2007; 35: 177-82.

20.- Sánchez J, Feris J, Fernández J, Pérez E,
Ramírez S, Ortega G, et al. Aislamiento de Klebsiella pneumoniae productora de betalactamasa de espectro extendido (BLEE) en recién nacidos en el Hospital Infantil Dr. Robert Reid Cabral de Santo Domingo, República Dominicana. Rev Panam Infectol 2005; 7: 15-20.

21.- Cordero L, Rau R, Taylor D, Ayers L. Enteric gram-negative bacilli bloodstream infections: 17 years' experience in a neonatal intensive care unit. Am J Infect Control 2004; 32: 189-95.

22.- Macias A, Muñoz J, Galván A, González J, Medinas H, Alpuche C, et al. Nosocomial bacteremia in neonates related to poor standards of care. Pediatr Infect Dis J 2005; 24: 713-6.

23.- Holmes A, Doré C, Saraswatula A, Bamford K, Richards M, Coello R, et al. Risk factors and recommendations for rate stratification for surveillance of neonatal healthcare-associated bloodstream infection. J Hosp Infect 2008; 68: 66-72.

24.- Richards C, Alonso J, Caicedo Y, Jarvis W. Klebsiella pneumoniae bloodstream infections among neonates in high-risk nursery in Cali, Colombia. Infect Control Hosp Epidemiol 2004; 25: 221-5. 